\section{Effect of pupil dilation on retinal nerve fibre layer thickness measurements and their repeatability with Cirrus HD-OCT}

G Savini ${ }^{1}$, M Carbonelli ${ }^{2}$, V Parisi ${ }^{1}$ and P Barboni ${ }^{2,3}$

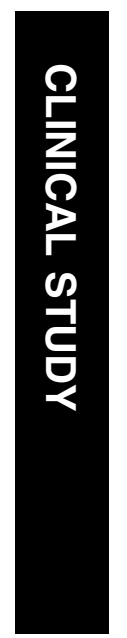

Eye (2010) 24, 1503-1508; doi:10.1038/eye.2010.66; published online 21 May 2010

Keywords: OCT; optic nerve; retinal nerve fibre layer

\section{Introduction}

Over the past decade, technologies measuring the peripapillary retinal nerve fibre layer (RNFL) thickness have gained wide popularity in ophthalmology. Optical coherence tomography (OCT), first described in 1991 by Huang et al, ${ }^{1}$ has a major role in this context and has been applied in the diagnosis of several diseases of the optic nerve, such as glaucoma, optic neuritis, papilledema, hereditary optic neuropathies, and multiple sclerosis. ${ }^{2-6}$ Until recently most clinical investigations were carried out by using time-domain OCT (TD-OCT), which has been available since the mid 90s on the OCT 1 and since 2002 on the Stratus OCT (both from Carl Zeiss Meditec, Dublin, CA, USA). TD-OCT uses a scanning interferometer and an 820-nm infrared light source that is split into two separate beams: one beam scans a tissue being analysed, and the other one functions as a reference beam that is reflected by a moving reference mirror. By comparing the two light beams, TD-OCT measures the optical backscattering of light to generate a cross-sectional image of the tested tissue. These instruments have been shown to provide measurements of the RNFL thickness with good repeatability and reproducibility, which are mandatory requisites for accurately assessing a disease and its progression. ${ }^{7-9}$ Moreover, their measurements have been
${ }^{1} \mathrm{~GB}$ Bietti Eye Foundation IRCCS, Rome, Italy

${ }^{2}$ Studio Oculistico d'Azeglio, Bologna, Italy

${ }^{3}$ Department of Neurological Sciences, University of Bologna, Bologna, Italy

Correspondence: G Savini, GB Bietti Eye Foundation IRCCS, Via Livenza 3,

Rome, Italy

Tel: + 390685356727 ;

Fax: + 390684242333

E-mail: giacomo.savini@ alice.it

Received: 29 September 2009

Accepted in revised form: 19 April 2010

Published online: 21 May 2010 
shown to correlate positively with ex vivo histological measurements of the retina. ${ }^{10,11}$

Spectral-domain OCT (SD-OCT) has now replaced TD-OCT and a large number of instruments by different manufacturers are commercially available. This technology provides increased axial resolution and scanning speed as it records the interferometric information using a spectrometric method instead of adjusting the position of a reference mirror. Hence, it does not need to measure reflectivity changes between retinal layers in each A-scan one at a time. Instead, the spectrometer detects the relative amplitudes of many optical frequencies simultaneously within the backscattered light. Many points are thus sampled at the same time, with all of the retinal layer depths of each A-scan calculated using Fourier transformation. ${ }^{12}$ Resolution in Fourier transformation is up to five times higher and imaging speed is up to 50 times faster than in conventional TD-OCT. ${ }^{13}$ Showing the accuracy of RNFL thickness measurements is an important first step towards validating latest-generation SD-OCTs for the diagnosis of glaucoma and glaucoma progression, as well as any other disease of the optic nerve.

Recently, several authors showed that the Cirrus HD-OCT has very good intra-observer repeatability in both healthy and glaucomatous eyes. ${ }^{14-16}$ Similar results were obtained by Gonzáles-García et $a l^{17}$ with another instrument (RTVue; Optovue Inc., Fremont, CA, USA).

Optic nerve and RNFL analyses by TD-OCT are usually carried out after dilating the pupil. Earlier studies using OCT1 and OCT 2 always followed this procedure, due to difficulties in visualizing the optic disc without pupil dilation. ${ }^{18-22}$ Recently, mydriasis was also induced using Stratus OCT. ${ }^{20,23-25}$ Some controversy, in fact, still exists regarding the effect of pupil dilation on RNFL thickness measurements by Stratus OCT: whereas some articles reported that the difference is little or even not statistically significant, ${ }^{26,27}$ other studies found that measurements before and after dilation are not interchangeable. ${ }^{28,29}$ Being able to acquire OCT scans without mydriasis would allow the operator to save time in a busy practice, would eliminate the risk of angle closure in predisposed eyes, and would not cause annoying visual disturbances in patients undergoing the examination. Moreover, it should be considered that during the follow-up it may occur that the same eye is examined sometimes with and without mydriasis.

Hence, the aim of this study was to assess whether pupil dilation affects RNFL thickness measurements by Cirrus HD-OCT and their repeatability.

\section{Materials and methods}

Between February and March 2009, 35 eyes of 35 subjects were enrolled in a private practice for this prospective observational case series. The study population consisted of consecutive patients with minor refractive disorders. All individuals underwent a complete ophthalmological examination, including visual acuity measurement, intraocular pressure measurement, slit-lamp biomicroscopy, and indirect ophthalmoscopy, to determine eligibility. Inclusion criteria were: best corrected visual acuity above $20 / 25$, refractive error between -3 and +3 dioptres of sphere or between -2 and +2 dioptres of cylinder, normal intraocular pressure $<21 \mathrm{~mm} \mathrm{Hg}$, normal appearance of the optic disc, no significant ocular disease found by routine ophthalmological examination, no history of glaucoma in the family, and/or systemic diseases with possible ocular involvement, such as diabetes mellitus.

Before pupil dilation, three individual $200 \times 200$ cube optic disc scans were obtained with Cirrus OCT (software version 3.0) as previously described by Knight et al. ${ }^{12}$ Once the subject was seated and properly aligned, the iris was brought into view. The scanning laser ophthalmoscopic image was focused by adjusting for refractive error. As the optic nerve head was centred on the live scanning laser ophthalmoscopic image using the internal fixation cross, centring (Z-offset) and enhancement (polarization) were optimized. A $6 \times 6 \mathrm{~mm}$ square of data was acquired. If the subject had an involuntary saccade during the scan, this was discarded and retaken (Figure 1). Ultimately, the signal strength had to be 6 or higher. Each optic disc scan captured a $6 \times 6 \times 2 \mathrm{~mm}$ 'cube' of data consisting of 200 A-scans from 200 linear B-scans (40000 points) in $1.5 \mathrm{~s}$ (27000 A-scans per second).

Because the data collected by Cirrus are a 'cube,' rather than a single circle as in TD-OCT, a comparable circle (radius $1.73 \mathrm{~mm}$ ) must be extracted from the data. In the Glaucoma Analysis, Cirrus HD-OCT algorithms find the optic disc, even if it is not well centred in the scan image,

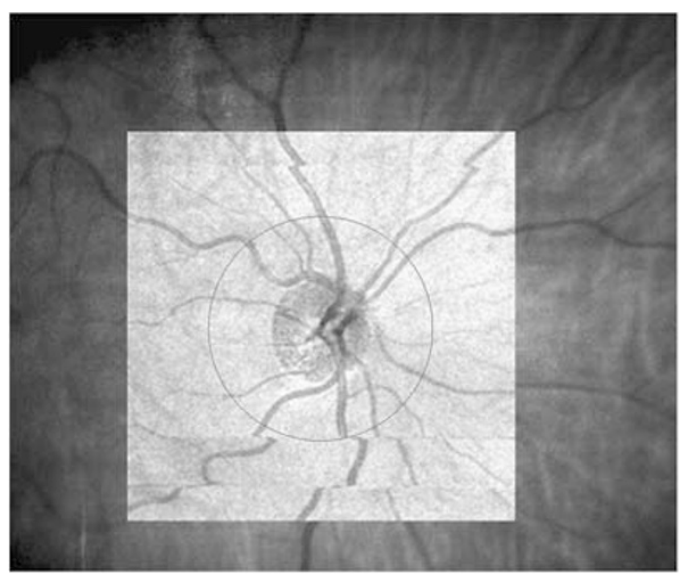

Figure 1 Typical example of eye movement artefact with vessel misalignment. Scans like this were excluded. 
and automatically place a calculation circle of $3.46 \mathrm{~mm}$ diameter evenly around it. Layer-seeking algorithms find the RNFL inner (anterior) boundary and RNFL outer (posterior) boundary for the entire cube, except the optic disc. The system extracts from the data cube 256 A-scan samples along the path of the calculation circle. The resulting temporal, superior, nasal, inferior, temporal profile map is equivalent to the Stratus peripapillary RNFL scan.

The same procedure was carried out after pupil dilation induced by $1 \%$ tropicamide.

All participants gave their informed consent. The study was conducted according to the Declaration of Helsinki Principles and was approved by the internal review board of the Department of Neurological Sciences at the University of Bologna.

\section{Statistics}

For the purposes of this study, the term repeatability was used according to the definition of the British Standards Institute, which considers it as a part of accuracy. ${ }^{30}$ Accuracy, as formally defined by BS ISO 5725-1, includes trueness and precision. Trueness is the inverse of bias and is obtained by comparing the measurement result with the accepted reference (conventional true) value. Precision is the inverse of statistical uncertainty, and is normally expressed in terms of standard deviation (SD). The factors involved include (1) the operator, (2) the equipment used, (3) the equipment calibration, (4) the environment, and (5) the elapsed time between measurements. Precision has two conditions: repeatability and reproducibility. Under repeatability conditions, factors such as $1-5$ are considered to be constant and do not contribute to the variability of the measurement result. Under reproducibility conditions, the factors can vary. Repeatability and reproducibility are the two extremes of precision.

We assessed repeatability by the following methods:

- Repeated-measures analysis of variance (ANOVA): this was automatically calculated using InStat software 3.0 (GraphPad, San Diego, CA, USA, 2003) for each of the RNFL parameters (average, four quadrants and 12 sectors) both before and after pupil dilation.

- Coefficient of variation (COV): this was calculated as the within-subject SD of the three repeated measurements divided by the within-subject mean, and was expressed as a percentage. . $^{8,31}$

- Intra-session test-retest variability in RNFL thickness: this was measured in micrometres and was calculated as twice the SD of the three repeated measurements for each measure of RNFL thickness. ${ }^{8}$

Differences between mean measurements before and after pupil dilation were assessed by a paired $t$-test.
We postulated that a mean difference of $3.5 \mu \mathrm{m}$ in the average RNFL thickness measurement could have been regarded as clinically significant, as this value corresponds to the intra-session test-retest variability reported by Budenz et $\mathrm{al}^{7}$ for Stratus OCT (data for Cirrus HD-OCT have not yet been published). Using PS version 3.0.12 (http:/ / biostat.mc.vanderbilt.edu/twiki/bin/ view/Main/PowerSampleSize, checked on 4 February 2010), we estimated a sample size of 29 eyes to detect such a difference with an SD of $\pm 4.4 \mu \mathrm{m}$ (corresponding to the SD of the difference between Stratus OCT average RNFL thickness measurements before and after mydriasis), ${ }^{26}$ and a power of $95 \%$ at a significant level of $5 \%$. A smaller sample would have been required to detect differences higher than $3.5 \mu \mathrm{m}$.

In all cases, differences were considered statistically significant when $P$-value was $<0.05$.

\section{Results}

Two patients were excluded, as the pupil diameter was too small to allow visualization of the optic nerve before pharmacological dilation. Another subject was excluded because of motion artefacts. Hence the statistical analysis was carried out on a final sample of 32 eyes (10 men and 22 women, mean age $59 \pm 19$ years) out of 35 .

Repeated-measures ANOVA did not show statistically significant differences among the three series of measurements either before or after mydriasis, in any of the quadrants and sectors, including the $360^{\circ}$ average measurement ( $P>0.05$ in all the cases). For each measurement, the mean of the three values did not show statistical differences before and after dilation, the only exception being the nasal sector at 9 o'clock, where the mean value was higher after mydriasis (Table 1).

On the basis of the results shown in Table 1, therefore, pupil dilation did not significantly affect RNFL thickness measurements by Cirrus HD-OCT. Nor did it affect the mean signal strength $(7.77 \pm 1.09$ before and $8.00 \pm 1.18$ after pupil dilation, $P>0.05)$.

As far as repeatability is concerned, excellent results were obtained for the COV and test-retest variability (Table 2).

\section{Discussion}

The present investigation shows that pupil dilation does not affect the RNFL thickness measurements provided by Cirrus HD-OCT: signal strength, mean values, and repeatability did not change before and mydriasis. Moreover, only in a small minority of subjects (2 out of $35,5.7 \%$ ) could the scan not be acquired because of an insufficient pupil diameter. These findings reveal noteworthy differences compared with the Stratus OCT. 
Table 1 Mean values of three measurements of the RNFL thickness before and after pupil dilation

\begin{tabular}{lccc}
\hline & $\begin{array}{c}\text { Mean thickness } \\
\pm \text { SD) before pupil } \\
\text { dilation }(\mu \mathrm{m})\end{array}$ & $\begin{array}{c}\text { Mean thickness } \\
( \pm \text { SD) after pupil } \\
\text { dilation }(\mu \mathrm{m})\end{array}$ & $\mathrm{P}$ \\
\hline $\begin{array}{l}\text { Average } \\
\text { Temporal }\end{array}$ & $88.53 \pm 11.10$ & $88.87 \pm 10.81$ & $\mathrm{NS}$ \\
quadrant & $59.19 \pm 7.8$ & $59.54 \pm 7.8$ & $\mathrm{NS}$ \\
$\begin{array}{l}\text { Superior } \\
\text { quadrant }\end{array}$ & $111.42 \pm 17.13$ & $111.68 \pm 16.65$ & $\mathrm{NS}$ \\
Nasal & $67.53 \pm 11.20$ & $68.10 \pm 10.65$ & $\mathrm{NS}$ \\
quadrant & & & \\
Inferior & $115.97 \pm 18.82$ & $115.73 \pm 19.35$ & $\mathrm{NS}$ \\
quadrant & & & \\
8 o'clock & $61.69 \pm 12.71$ & $62.04 \pm 13.22$ & $\mathrm{NS}$ \\
9 o'clock & $46.35 \pm 6.46$ & $47.38 \pm 6.55$ & 0.0059 \\
10 o'clock & $69.43 \pm 9.55$ & $69.21 \pm 8.95$ & $\mathrm{NS}$ \\
11 o'clock & $118.83 \pm 19.58$ & $118.05 \pm 20.07$ & $\mathrm{NS}$ \\
12 o'clock & $115.11 \pm 29.15$ & $116.50 \pm 28.23$ & $\mathrm{NS}$ \\
1 o'clock & $100.32 \pm 24.65$ & $101.37 \pm 25.05$ & NS \\
2 o'clock & $81.70 \pm 14.85$ & $81.71 \pm 14.27$ & NS \\
3 o'clock & $57.31 \pm 10.11$ & $57.86 \pm 10.69$ & NS \\
4 o'clock & $63.63 \pm 14.07$ & $64.75 \pm 12.66$ & NS \\
5 o'clock & $94.91 \pm 27.80$ & $95.69 \pm 27.20$ & NS \\
6 o'clock & $129.54 \pm 28.09$ & $128.85 \pm 28.87$ & NS \\
7 o'clock & $123.41 \pm 17.13$ & $122.68 \pm 18.57$ & NS \\
\hline
\end{tabular}

Table 2 COV and test-retest variability of intra-session RNFL thickness measurements with Cirrus HD-OCT compared to intra-session values obtained by Budenz et $a l^{7}$ with TD-OCT and a standard $3.4 \mathrm{~mm}$ scan

\begin{tabular}{|c|c|c|c|c|c|c|}
\hline \multirow{2}{*}{$\begin{array}{l}\text { Quadrant } \\
\text { Mydriasis }\end{array}$} & \multicolumn{3}{|c|}{$\mathrm{COV}$} & \multicolumn{3}{|c|}{ Test-retest variability } \\
\hline & No & Yes & $T D-O C T^{7}$ & No & Yes & $T D-O C T^{7}$ \\
\hline Average & 1.37 & 1.36 & 1.7 & 2.39 & 2.41 & 3.5 \\
\hline Temporal & 1.84 & 2.18 & 5.1 & 2.17 & 2.55 & 7.5 \\
\hline Superior & 2.47 & 2.29 & 3.8 & 5.42 & 5.17 & 9.6 \\
\hline Nasal & 2.97 & 2.62 & 6.7 & 4.16 & 3.60 & 10.2 \\
\hline Inferior & 2.27 & 2.43 & 3.7 & 5.00 & 5.62 & 9.7 \\
\hline 8 o'clock & 3.16 & 2.82 & - & 3.96 & 3.49 & - \\
\hline 9 o'clock & 2.49 & 2.92 & - & 2.27 & 2.73 & - \\
\hline 10 o'clock & 2.07 & 2.85 & - & 2.84 & 3.92 & - \\
\hline 11 o'clock & 2.85 & 3.46 & - & 7.02 & 8.14 & - \\
\hline 12 o'clock & 3.69 & 3.75 & - & 8.40 & 8.28 & - \\
\hline 1 o'clock & 3.54 & 3.56 & - & 7.03 & 7.30 & - \\
\hline 2 o'clock & 4.46 & 4.48 & - & 7.53 & 7.32 & - \\
\hline 3 o'clock & 3.65 & 3.46 & - & 4.22 & 4.15 & - \\
\hline 4 o'clock & 4.10 & 3.60 & - & 5.18 & 4.60 & - \\
\hline 5 o'clock & 4.33 & 4.17 & - & 7.46 & 7.85 & - \\
\hline 6 o'clock & 3.70 & 3.62 & - & 9.18 & 9.29 & - \\
\hline 7 o'clock & 3.00 & 3.04 & - & 7.35 & 7.40 & - \\
\hline
\end{tabular}

In a study involving 38 glaucoma patients with a mean age similar to that in our own sample, Smith et al. ${ }^{28}$ reported that in almost a quarter of cases (23.7\%) it was not possible to acquire high-quality OCT images without pupil dilation and that dilated scans were more reproducible and gave different RNFL thickness mean values. A significant effect of pupil dilation was found also by Paunescu et al. ${ }^{29}$ In a previous study on a group of patients undergoing cataract surgery, our group reported that pupil dilation slightly affected RNFL thickness measurements by Stratus OCT, as RNFL thickness values were statistically higher in the $360^{\circ}$ average measurement and in the nasal quadrant and some differences were found in the other quadrants as well, although statistical difference was not reached. ${ }^{26}$ Just one study found that pupil dilation did not induce any statistical difference in RNFL thickness measurements, but the sample of this investigation was probably too small (10 eyes) to detect such a difference. ${ }^{27}$ Overall, therefore, we can conclude that SD-OCT is less influenced than TD-OCT by pupil size and this represents a further step forward for this technology.

Our study results also confirm the excellent repeatability of Cirrus HD-OCT RNFL thickness measurements and suggest that the measurements provided by Cirrus HD-OCT and RTVue have similar repeatability. ${ }^{14-17}$ This is a mandatory requirement of any instrument designed not only to diagnose, but also to enable a follow-up assessment of possible changes in any measurement. If there is excellent repeatability, with little variance in measurements, then identifying small changes in those measurements over time will be meaningful, as long as one accounts for age-related changes. Conversely, in the case of poor repeatability, relatively large pathological changes will be needed before true progression of the disease may be diagnosed. ${ }^{8}$

Compared to the intra-session repeatability of TD-OCT as reported in previous studies, the repeatability achieved using Cirrus HD-OCT proved to be higher. As shown in Table 2, Cirrus HD-OCT had lower COVs and test-retest variability compared with Stratus OCT, based on the data reported by Budenz et al. ${ }^{7}$ The most likely explanation for the better performance of Cirrus HD-OCT is related to the position of the circular scan: Cirrus HD-OCT does not depend on the operator correctly placing the scan because it includes an automated graph-based algorithm (AutoCenter) that identifies centre and border of the optic disc in peripapillary images. In contrast, the Stratus OCT requires the operator to choose the location of the circle, and previous studies have already shown that this procedure may affect RNFL thickness measurements. ${ }^{32,33}$

Interestingly, we also confirmed that RNFL thickness measurements are slightly less repeatable in the nasal quadrant. This finding has already been reported with both TD-OCT and SD-OCT, ${ }^{14}$ and, as previously suggested, may be related to the fact that the angle of incidence of the illuminating beam is such that the RNFL is dimmer nasally, so that the edges of the RNFL are less reliably identified. ${ }^{8}$ 
This study did not address two important issues: repeatability in glaucomatous eyes and both interobserver and inter-visit reproducibility. Although the former evaluation has been recently carried out (with excellent results), ${ }^{14}$ the latter will be the subject of further investigations.

In conclusion, our study data confirm that Cirrus HD-OCT measurements of the RNFL thickness show excellent repeatability, on condition that no artefacts are produced during the scan acquisition, and are not influenced by pupil size.

\section{Summary}

\section{What was known before}

- Spectral-domain OCT provides faster scanning of the retina and optic nerve than time-domain OCT.

\section{What this study adds}

- Spectral-domain OCT measurements of the retinal nerve fibre layer thickness provide also the highest repeatability, with and without pupil dilation.

\section{Conflict of interest}

The authors declare no conflict of interest.

\section{References}

1 Huang D, Swanson EA, Lin CP, Schuman JS, Stinson WG, Chang W et al. Optical coherence tomography. Science 1991; 254: 1178-1181.

2 Lee EJ, Kim TW, Park KH, Seong M, Kim H, Kim DM. Ability of Stratus OCT to detect progressive retinal nerve fiber layer atrophy in glaucoma. Invest Ophthalmol Vis Sci 2009; 50: 662-668.

3 Lu AT, Wang M, Varma R, Schuman JS, Greenfield DS, Smith SD et al. Advanced Imaging for Glaucoma Study Group. Combining nerve fiber layer parameters to optimize glaucoma diagnosis with optical coherence tomography. Ophthalmology 2008; 115: 1352-1357.

4 Savini G, Bellusci C, Carbonelli M, Zanini M, Carelli V, Sadun AA et al. Detection and quantification of retinal nerve fiber layer thickness in optic disc edema using stratus OCT. Arch Ophthalmol 2006; 124: 1111-1117.

5 Barboni P, Savini G, Valentino ML, Montagna P, Cortelli P, De Negri AM et al. Retinal nerve fiber layer evaluation by optical coherence tomography in Leber's hereditary optic neuropathy. Ophthalmology 2005; 112: 120-126.

6 Siger M, Dziegielewski K, Jasek L, Bieniek M, Nicpan A, Nawrocki J et al. Optical coherence tomography in multiple sclerosis: thickness of the retinal nerve fiber layer as a potential measure of axonal loss and brain atrophy. J Neurol 2008; 255: 1555-1560.

7 Budenz DL, Chang RT, Huang X, Kinghton RW, Tielsch JM. Reproducibility of retinal nerve fiber thickness measurements using the Stratus OCT in normal and glaucomatous eyes. Invest Ophthalmol Vis Sci 2005; 46: 2440-2443.
8 Budenz DL, Fredette MJ, Feuer WJ, Anderson DR. Reproducibility of peripapillary retinal nerve fiber thickness measurements with stratus OCT in glaucomatous eyes. Ophthalmology 2008; 115: 661-666.

9 Gürses-Özden R, Ten C, Vessani R, Zafar S, Liebmann JS, Ritch R. Macular and retinal nerve fiber layer thickness measurement reproducibility using optical coherence tomography (OCT-3). J Glaucoma 2004; 13: 238-244.

10 Schuman JS, Pedut-Kloizman T, Pakter H, Wang N, Guedes $\mathrm{V}$, Huang $\mathrm{L}$ et al. Optical coherence tomography and histologic measurements of nerve fiber layer thickness in normal and glaucomatous monkey eyes. Invest Ophthalmol Vis Sci 2007; 48: 3645-3654

11 Blumenthal EZ, Parikh RS, Pe'er J, Naik M, Kaliner E, Cohe MJ et al. Retinal nerve fibre layer imaging compared with histological measurements in a human eye. Eye 2009; 23: 171-175.

12 Knight OJ, Chang TR, Feuer WJ, Budenz DL. Comparison of retinal nerve fiber layer measurements using time domain and spectral domain optical coherence tomography. Ophthalmology 2009; 116: 1271-1277.

13 Wojtkowski M, Leitgeb R, Kowalczyk A, Bajraszewski T, Fercher AF. In vivo human retinal imaging by Fourier domain optical coherence tomography. J Biomed Opt 2002; 7: 457-463.

14 Vizzeri G, Weinreb RN, Gonzáles-García AO, Bowd C, Medeiros FA, Sample PA et al. Agreement between spectraldomain and time-domain OCT for measuring RNFL thickness. Br J Ophthalmol 2009; 93: 775-781.

15 Leung CK, Cheung CY, Weinreb RN, Qui Q, Liu S, Li H et al. Retinal nerve fiber layer imaging with spectral-domain optical coherence tomography: a variability and diagnostic performance study. Ophthalmology. 2009; 116: 1257-1263.

16 Sung KR, Kim DY, Park SB, Kook MS. Comparison of retinal nerve fiber layer thickness measured by Cirrus HD and Stratus optical coherence tomography. Ophthalmology 2009; 116: $1264-1270$.

17 Gonzáles-García AO, Vizzeri G, Bowd C, Medeiros FA, Zangwill LM, Weinreb RN. Reproducibility of RTVue retinal nerve fiber layer thickness and optic disc measurements and agreement with Stratus Optical Coherence Tomography measurements. Am J Opthalmol 2009; 147: 1067-1074.

18 Sugimoto M, Ito K, Goto R, Uji Y. Symmetry analysis for detecting early glaucomatous changes in ocular hypertension using optical coherence tomography. Jpn J Ophthalmol 2004; 48: 281-286.

19 Varma R, Bazzaz S, Lai M. Optical tomography-measured retinal nerve fiber layer thickness in normal Latinos. Invest Ophthalmol Vis Sci 2003; 44: 3369-3373.

20 Bourne RRA, Medeiros FA, Bowd C, Jahanbakhsh K, Zangwill LM, Weinreb RN. Comparability of retinal nerve fiber layer thickness measurements of optical coherence tomography instruments. Invest Ophthalmol Vis Sci 2005; 46: $1280-1285$.

21 Alamouti B, Funk J. Retinal thickness decreases with age: an OCT study. Br J Ophthalmol 2003; 87: 899-901.

22 Carpineto P, Ciancaglini M, Zuppardi E, Falconio G, Doronzo E, Mastropasqua L. Reliability of nerve fiber layer thickness measurements using optical coherence tomography in normal and glaucomatous eyes. Ophthalmology 2003; 110: 190-195.

23 Kim TW, Kim T-W, Park KH, Kim DM. An unexpectedly low Stratus optical coherence tomography false-positive rate in the non-nasal quadrants of Asian eyes: indirect 
evidence of differing retinal nerve fibre layer thickness profiles according to ethnicity. Br J Ophthalmol 2008; 92: 735-739.

24 Chen HY, Huang ML. Discrimination between normal and glaucomatous eyes using Stratus optical coherence tomography in Taiwan Chinese subjects. Graefes Arch Clin Exp Ophthalmol 2005; 243: 894-902.

25 Huang ML, Chen HY, Lin JC. Rule extraction for glaucoma detection with summary data from StratusOCT. Invest Ophthalmol Vis Sci 2007; 48: 244-250.

26 Savini G, Zanini M, Barboni P. Influence of pupil size and cataract on retinal nerve fiber layer thickness measurements by Stratus OCT. J Glaucoma 2006; 15: 336-340.

27 Zafar S, Gurses-Ozden R, Vessani R, Zafar S, Liebmann JM, Ritch R. Effect of pupillary dilation on retinal nerve fiber layer thickness measurements using optical coherence tomography. J Glaucoma 2004; 13: 34-37.

28 Smith M, Frost A, Graham CM, Shaw S. Effect of pupillary dilatation on glaucoma assessments using optical coherence tomography. $\mathrm{Br} J$ Ophthalmol 2007; 91: 1686-1690.

29 Paunescu LA, Schuman JS, Price LL, Starck PC, Beaton S, Ishikawa $\mathrm{H}$ et al. Reproducibility of nerve fiber thickness, macular thickness, and optic nerve head measurements using StratusOCT. Invest Ophthalmol Vis Sci 2004; 45: 1716-1724.

30 British Standards Institution. BS ISO 5725-1:1994 Accuracy (Trueness and Precision) of Measurement Methods and Results - Part1: General Principles and Definitions. BSI: London, 1994, pp 1-26.

31 Mohamed S, Lee GKY, Rao SK, Wong Al, Cheng AC, li EY et al. Repeatability and reproducibility of pachymetric mapping with Visante anterior segment-optical coherence tomography. Invest Ophthalmol Vis Sci 2007; 48: 5499-5504.

32 Gabriele ML, Ishikawa H, Wollstein G, Bilonick RA, Townsend KA, Kagemann L et al. Optical coherence tomography scan circle location and mean retinal nerve fiber layer measurement variability. Invest Ophthalmol Vis Sci 2008; 49: 2315-2321.

33 Vizzeri G, Bowd C, Medeiros FA, Weinreb RN, Zangwill LM. Effect of improper scan alignment on retinal nerve fiber layer thickness measurements using Stratus optical coherence tomograph. J Glaucoma 2008; 17: 341-349. 